\title{
Capacity and Outage Analysis of MIMO and Cooperative Communication Systems in Underground Tunnels
}

\author{
Zhi Sun, Student Member, IEEE, Ian F. Akyildiz, Fellow, IEEE, and \\ Gerhard P. Hancke, Senior Member, IEEE
}

\begin{abstract}
In underground mine and road tunnels, multipath fading is much more severe than the terrestrial wireless channels. To overcome the multipath fading in underground tunnels, MIMO (Multiple Input Multiple Output) and Cooperative Communication system can be utilized. Since the underground channel characteristics are significantly different from those in terrestrial environments, the channel capacity and the outage behavior of such systems need to be investigated based on underground tunnel channel models, which has not been addressed by the research community yet. In this paper, the capacity distribution and outage probability of MIMO and cooperative communication systems are investigated in underground tunnel environments. Explicit formulas of the capacity distribution and outage probability are developed as functions of environmental conditions and system configurations. Based on the capacity and outage analysis in underground tunnels, the optimal MIMO antenna geometry design scheme is proposed for MIMO systems; and the cooperative relay assignment protocol is developed for cooperative communication systems. Simulations are conducted to validate the theoretical results.
\end{abstract}

\section{Index Terms}

Underground Mine Tunnel, Underground Road Tunnel, MIMO, Cooperative Communications, Channel Capacity, Outage Behavior, MIMO Antenna Geometry, Cooperative Relay Assignment.

\section{INTRODUCTION}

Wireless communication networks and wireless sensor networks are expected to improve the safety and the productivity/efficiency in underground mine tunnels and road tunnels [1]. In these wireless networks, high link reliability is required to guarantee the personal and property safety in

Zhi Sun and Ian F. Akyildiz are with the Broadband Wireless Networking Laboratory, School of Electrical and Computer Engineering, Georgia Institute of Technology, Atlanta, GA, 30332, United States. E-mail: \{zsun; ian\}@ece.gatech.edu

Gerhard P. Hancke is with Department of Electrical, Electronic \& Computer Engineering University of Pretoria, South Africa, 0002. E-mail: g.hancke@ieee.org 
the high-risky underground tunnel environments. However, the link error caused by the multipath fading in underground tunnels is much more severe than the terrestrial wireless channels, since the wireless signals are confined to the internal space of the underground mines and tunnels [2], [3]. Moreover, the reflections and the diffractions on the mobile vehicles or machineries inside the tunnels create even more severe multipath fading [4], [5].

To solve the unreliable link problem caused by the multipath fading in underground tunnels, spatial diversity-based techniques including MIMO (Multiple Input Multiple Output) and Cooperative Communication system can be utilized. In particular, the MIMO system employs multiple antenna elements at both transmitter and receiver to achieve the spatial diversity [12], which is suitable for large devices such as the base stations and the mobile terminals on vehicles. In contrast, the cooperative communication system [13] explores the broadcast nature of the wireless channel and utilize multiple wireless nodes with single antenna to form a virtual MIMO, which is suitable for small and low-cost devices such as wireless sensors and handsets.

As the most important criteria in designing MIMO and cooperative communication systems, the channel capacity and the outage behavior need to be investigated in underground tunnel environments. Specifically, according to our previous research on wireless channels in tunnel environments [2], [3], [4], [5], the channel characteristics in underground tunnels are significantly different from the terrestrial channel. First, due to the reflections on the tunnel walls, the propagation of electromagnetic (EM) waves form regular patterns (i.e. modes) in underground tunnels. Each propagation mode has different field distribution and attenuation rate. The effectiveness of excitation and reception of those modes is determined by the position of the transmitter and the receiver [2], [3]. Second, obstructions with random sizes and positions inside the tunnel, such as vehicles and machineries, cause additional loss and coupling of the propagation modes [4], [5]. Since the channel characteristics have straightforward influences on the channel capacity, it is necessary to analyze the capacity and outage behavior of the MIMO and cooperative communication systems based on the unique channel model in the underground tunnels, which have not been addressed by the research community so far, to the best of our knowledge.

In this paper, the capacity distribution and outage probability of MIMO and cooperative communication systems in underground tunnels are investigated. In particular, for MIMO system in underground tunnels, based on the underground channel model, we prove that the MIMO capacity in underground tunnels follows either a normal distribution in high signal-to-noise ratio (SNR) regime or a log-normal distribution in low SNR regime. The parameters of each distribution are explicitly provided. The ergodic capacity and outage capacity of MIMO system are calculated after the capacity distribution is derived. Then, to maximize the outage capacity, the optimal MIMO antenna geometry is designed in both high SNR regime and low SNR regime. For cooperative communication system in underground tunnels, the outage probability are calculated by utilizing the tunnel channel model. Then a fully distributed optimal relay assignment protocol 
in underground tunnels is developed to minimize the outage probability. Finally, the theoretical analysis is validated by simulations in various environmental and system conditions.

The remainder of this paper is organized as follows. In Section II, the related work is introduced. In Section III, the channel model in underground tunnels is overviewed. Next, in Section IV, the capacity distribution and the outage capacity of MIMO system in underground tunnels are analyzed. The optimal MIMO antenna geometry is then designed based on the capacity and outage analysis. After that, in Section V, the outage probability of the cooperative communication system in underground tunnels are calculated, based on which the optimal relay assignment assignment protocol is developed. In Section VI, simulation evaluations are provided. Finally, the paper is concluded in Section VII.

\section{RELATED WORKS}

In the literature, there are mainly three types of models to characterize the wireless channel in empty tunnels [6]: the Geometrical Optical model (GO model) [7], [8], the Waveguide model [9], [10], and the Full Wave model [11]. The GO model and the Full Wave model can only provide numerical results and the computational complexity is high. Although the waveguide model can provide analytical results, it is only applicable for the far region of the transmitter in tunnels. In [3], [2], we have developed the Multimode model that gives analytical results for both the near region and the far region in an empty tunnel. Moreover, in [4], [5], we extended the Multimode model so that the signal propagation in tunnels with vehicular traffic flow can be analytically modeled.

The MIMO capacity has been widely analyzed in terrestrial wireless communication systems. In [12] and [14], the MIMO capacity over the additive Gaussian channel with and without multipath fading is analyzed. In [15], the effect of MIMO antenna geometry on capacity is analyzed to mitigate the impact of the correlated MIMO channel. In [16], it is proved that the distribution of the MIMO capacity in terrestrial channel follows a normal distribution under the condition that the number of antenna elements is large. All the above works are based on the terrestrial wireless channel model that is simpler and fuzzier than the tunnel channel model in two aspects: 1) The channel gain of the terrestrial channel is assumed to be a Rayleigh random variable multiplied by a power function of the transmission distance. However, the parameters of the Rayleigh fading cannot be accurately calculated. In contrast, the channel gain in underground tunnels is a weighted sum of multiple propagation modes. The intensity and the field distribution of each mode can be accurately characterized. 2) In terrestrial channel, each pair of TX and RX antenna elements in the MIMO system is assumed to have the same mean channel gain. However, in underground tunnels, the positions of the transceivers have significant influences on the channel gain. Hence the MIMO antenna geometry significantly affects MIMO channel capacity in tunnels. 
In [17], current terrestrial MIMO techniques are evaluated in tunnel environments by simulations. It shows that the MIMO technique can also effectively mitigate multipath fading in underground tunnels. In [18], [19], the MIMO channel capacity in empty waveguide and cavity channels are calculated using the modal expansion technique. Those existing works on MIMO capacity in tunnels are based on the empty tunnel channel model. However, in real underground tunnels, there are a large number of random obstructions, such as vehicles and mining machines. Hence the randomness of the MIMO capacity caused by the random obstructions has significant influence on the performance of the MIMO systems in underground tunnels. To the best knowledge of the authors, the capacity distribution as well as the outage behavior of the MIMO systems in tunnels have not been investigated yet.

The cooperative communication technique has also been intensively investigated in terrestrial environments recently. In [20], several efficient cooperative schemes are proposed, and the corresponding outage behavior in terms of outage probability are investigated in high SNR regime. In [21], the outage capacity of cooperative communication system is calculated in low SNR regime. In [22], a centralized cooperative relay assignment protocol is proposed to maximize the minimum cooperative capacity in the whole network. In [23], a distributed relay assignment protocols are proposed. However, it still requires the information exchanges among the source node, relay nodes and destination node, which is difficult to achieve in networks with high dynamic topology, such as the vehicular networks in road tunnels. In [24], a nearest neighbor relay assignment protocol is proposed based on the analysis on the outage probability, which is fully distributed and only requires the local position information. Similar to the existing works on MIMO system, the above works on cooperative communications are also based on the terrestrial wireless channel. Currently, there is no existing paper on either the outage analysis or the relay assignment for cooperative communications in underground tunnels.

In this paper, we analyze the capacity distribution and outage behavior of the MIMO and cooperative communication system in underground tunnel environments. The parameters of the capacity distribution and the outage probability are explicitly expressed as functions of the tunnel environmental conditions, the antenna geometry (for MIMO), and the relay assignment strategy (for cooperative communications). Then, based on the capacity and outage analysis, the optimal MIMO antenna geometry and the optimal cooperative relay assignment protocol are developed for wireless communications in underground tunnels.

\section{Channel Model in Underground Tunnels}

\section{A. Channel Model for Empty Tunnels}

Multiple modes propagate simultaneously in empty tunnels. The tunnel cross section is treated as an equivalent rectangular with a width of $2 a \mathrm{~m}$ and a height of $2 b \mathrm{~m}$. A Cartesian coordinate system is set with its origin located at the center of the rectangle tunnel, as shown in Fig. 1. 
By solving Maxwell's equations using the boundary conditions, multiple solutions of the EM field distribution in the tunnel can be derived. Each solution refers to a propagation mode and has different EM field distribution and attenuation rate [25]. The EM field distribution of each mode in the tunnel cross section is characterized by its eigenfunction [3]:

$$
E_{m n,(x, y)}^{e i g n} \simeq \sin \left(\frac{m \pi}{2 a} x+\varphi_{x}\right) \cdot \cos \left(\frac{n \pi}{2 b} y+\varphi_{y}\right)
$$

where $x$ and $y$ are the position coordinates in the tunnel cross section; $\varphi_{x}=0$ if $m$ is even; $\varphi_{x}=\frac{\pi}{2}$ if $m$ is odd; $\varphi_{y}=0$ if $n$ is odd; $\varphi_{y}=\frac{\pi}{2}$ if $n$ is even; $m$ and $n$ indicate the mode order, where lower order modes have lower attenuation rates. The intensity of each mode can be derived by a mode matching technique given in [3].

Let $\mathbf{N}_{\text {mode }}$ be the set of modes that have significant energy, $\mathbf{N}_{\text {mode }}=\{(m, n)\},\left|\mathbf{N}_{\text {mode }}\right|=N$. The complex channel gain $h_{i j}$ between receiver $i$ and transmitter $j$ inside the tunnel can be obtained by summing up the gains of all significant modes at receiver's position [3]:

$$
h_{i j}=\sqrt{G_{t} G_{r}} \cdot \sum_{(m, n) \in \mathbf{N}_{\text {mode }}} E_{m n,\left(x_{i}, y_{i}\right)}^{e i g n} \cdot C_{m n, j}^{T X} \cdot e^{-\Gamma_{m n} \cdot z_{r}},
$$

where $z_{r}$ is the distance between the transmitter and the receiver; $\left(x_{i}, y_{i}\right)$ is the coordinates on the tunnel cross section of the receiver $i ; G_{t}$ and $G_{r}$ are the TX and RX antenna gain, respectively; $\Gamma_{m n}$ is the attenuation coefficient of the $E H_{m n}$ mode (the detailed expression of $\Gamma_{m n}$ can be found in [2], [3]); $E_{m n,(x, y)}^{e i g n}$ is the value of the eigenfunction given in (1); $C_{m n, j}^{T X}$ is the intensity of the $E H_{m n}$ mode near the transmitter $j . C_{m n, j}^{T X}$ is derived in [3], which is:

$$
C_{m n, j}^{T X}=\frac{\pi}{a b \sqrt{1-\left(\frac{m \pi}{2 a k}\right)^{2}-\left(\frac{n \pi}{2 b k}\right)^{2}}} \cdot E_{m n,\left(x_{j}, y_{j}\right)}^{e i g n},
$$

where $k$ is the wave number; $\left(x_{j}, y_{j}\right)$ are the coordinates of the transmitter $j$.

\section{B. Channel Model for Tunnels with Obstructions}

Actual tunnels are filled with obstructions with different sizes and positions. Since small obstructions do not have significant influence on the signal propagation in tunnels, the obstructions considered here are refereed to traffic flow of vehicles or machineries inside the tunnels. In [4], [5], we have theoretically modeled the influence of the traffic flow on the signal propagation in tunnels. Consider that a traffic flow with $M$ vehicles exists between the transceivers in the tunnel, as shown in Fig. 1. The position and size of the $k^{t h}$ vehicle are $\left(x_{v}^{k}, z_{v}^{k}\right)$ and $\left(w_{k}, h_{k}, l_{k}\right)$, respectively. According to US Federal Regulations, the width $w_{k}$, height $h_{k}$ and length $l_{k}$ of most vehicles (including cars, vans, buses and trucks) fall into the following intervals (unit is meter): $w_{k} \in[1.5,2.5], h_{k} \in[1.3,4.2]$, and $l_{k} \in[3.5,16.2]$.

The transmitter is located at $z_{0}=0 \mathrm{~m}$ and the receiver is located ate $z_{r}$. Then according to 
[4], [5], the channel gain $h_{i j}$ between the transmitter $j$ and the receiver $i$ can be expressed as

$$
h_{i j}=\sqrt{G_{t} G_{r}} \cdot \mathbf{E}_{\left(x_{i}, y_{i}\right)}^{e i g n} \cdot \mathbf{A}\left(z_{r}-z_{M}\right) \cdot \prod_{k=1}^{M}\left[\mathbf{B}_{k} \cdot \mathbf{A}\left(z_{k}-z_{k-1}\right)\right] \cdot \mathbf{C}_{\left(x_{j}, y_{j}\right)}^{T X}
$$

where $\mathbf{E}_{\left(x_{i} y_{i}\right)}^{\text {eign }}$ is the eigenfunction vector of the receiver $i$ at $\left(x_{i}, y_{i}, z_{r}\right)$; and $\mathbf{C}_{\left(x_{j} y_{j}\right)}^{T X}$ is the mode intensity vector of the transmitter $j$ at $\left(x_{j}, y_{j}, z_{0}\right)$ :

$$
\mathbf{E}_{\left(x_{i} y_{i}\right)}^{e i g n}=\left[E_{1,\left(x_{i}, y_{i}\right)}^{e i g n}, E_{2,\left(x_{i}, y_{i}\right)}^{e i g n}, \cdots E_{N,\left(x_{i}, y_{i}\right)}^{e i g n}\right], \quad \mathbf{C}_{\left(x_{j} y_{j}\right)}^{T X}=\left[C_{1,\left(x_{j}, y_{j}\right)}^{T X}, C_{2,\left(x_{j}, y_{j}\right)}^{T X} \cdots C_{N,\left(x_{j}, y_{j}\right)}^{T X}\right] ;
$$

$\mathbf{A}(z)$ is the $N \times N$ attenuation matrix of transmitting all the $N$ modes for $z$ meters in an empty tunnel; and $\mathbf{B}_{k}$ is the $N \times N$ influence matrix caused by the $k^{t h}$ vehicle in the traffic flow:

$$
\mathbf{A}(z)=\left(\begin{array}{cccc}
e^{-\Gamma_{1} \cdot z} & 0 & \cdots & 0 \\
0 & e^{-\Gamma_{2} \cdot z} & \cdots & 0 \\
\vdots & & \ddots & \vdots \\
0 & 0 & \cdots & e^{-\Gamma_{N} \cdot z}
\end{array}\right), \quad \mathbf{B}_{k}=\left(\begin{array}{cccc}
1-L_{1}^{k} & B_{2 \rightarrow 1}^{k l} & \cdots & B_{N \rightarrow 1}^{k} \\
B_{1 \rightarrow 2}^{k} & 1-L_{2}^{k} & \cdots & B_{N \rightarrow 2}^{k} \\
\vdots & \vdots & \ddots & \vdots \\
B_{1 \rightarrow N}^{k} & B_{2 \rightarrow N}^{k} & \cdots & 1-L_{N}^{k}
\end{array}\right) \text {, }
$$

where $L_{m n}^{k}$ on the diagonal of the matrix is the additional loss of the $E H_{m n}$ mode caused by the $k^{\text {th }}$ vehicle in the traffic flow:

$$
L_{m n}^{k}=\frac{1}{4 a b}\left[w_{k}-\frac{2 a}{m \pi}(-1)^{m} \cos \left(\frac{m \pi}{a} x_{v}^{k}\right) \sin \left(\frac{m \pi}{a} w_{k}\right)\right] \cdot\left[h_{k}-\frac{b}{n \pi} \sin \left(\frac{n \pi}{b} h_{k}\right)\right] ;
$$

Except the elements on the diagonal of the matrix $\mathbf{B}_{k}$, the other elements, i.e. $B_{m n \rightarrow s t}^{k}$, are the mode coupling coefficients. According to [4], [5], the influence matrix $\mathbf{B}_{k}$ can be approximately viewed as a diagonal matrix since the mode coupling coefficients are much smaller than the elements on the matrix diagonal. Consequently, the channel gain $h_{i j}$ in (4) can be simplified as

$$
h_{i j}=\sqrt{G_{t} G_{r}} \cdot \mathbf{E}_{\left(x_{i}, y_{i}\right)}^{e i g n} \cdot \mathbf{D}_{z_{r}}^{M} \cdot \mathbf{C}_{\left(x_{j}, y_{j}\right)}^{T X},
$$

where $\mathbf{D}_{z_{r}}^{M}$ is the propagation matrix given by

$$
\mathbf{D}_{z_{r}}^{M}=\left(\begin{array}{ccc}
e^{-\Gamma_{1} z_{r}} \cdot \prod_{k=1}^{M}\left(1-L_{1}^{k}\right) & \cdots & 0 \\
\vdots & \ddots & \vdots \\
0 & \cdots & e^{-\Gamma_{N} z_{r} \cdot} \prod_{k=1}^{M}\left(1-L_{N}^{k}\right)
\end{array}\right)
$$

\section{CApacity And Outage Behavior of MiMO Systems in Underground Tunnels}

In this section, the capacity and the outage behavior of the MIMO systems are investigated based on the underground tunnel channel model provided in Section III. Explicit formulas of capacity probability density function (PDF), ergodic capacity, and outage capacity are developed. Based on the analysis of MIMO capacity and outage behavior, an outage-optimal MIMO antenna geometry design scheme is developed to maximize the MIMO outage capacity. 


\section{A. MIMO Capacity in Underground Tunnels}

We consider a narrowband channel with $p$ transmitting (TX) and $q$ receiving antenna elements. The complex channel gain matrix $\mathbf{H}$ is a $q \times p$ matrix $\left[h_{i j}\right]_{q \times p}$. The matrix element $h_{i j}$ is the channel gain between RX antenna $i$ and TX antenna $j$, which is given in (8). We assume that the transmitter does not have the channel state information (CSI). Hence, equal power is allocated to each TX antenna. The MIMO capacity with equal power allocation is given by [12]

$$
\mathbb{C}_{\text {MIMO }}=E\left[\log \operatorname{det}\left(\mathbf{I}_{q \times q}+\rho / p \cdot \mathbf{H} \cdot \mathbf{H}^{*}\right)\right],
$$

where $\rho$ is the signal to noise ratio (SNR) at the transmitter, which is defined as $\rho=\frac{P_{t o t}}{N_{0}}$, where $P_{t o t}$ is the total transmission power of all TX antenna elements; $N_{0}$ is the noise power. The channel gain matrix $\mathbf{H}$ can be derived from (8):

$$
\mathbf{H}=\sqrt{G_{t} G_{r}} \cdot \mathbf{E}^{R X} \cdot \mathbf{D}_{z_{r}}^{M} \cdot \mathbf{C}^{T X},
$$

where $\mathbf{D}^{M}\left(z_{r}\right)$ is the propagation matrix defined in (9); $\mathbf{E}^{R X}$ is the mode eigenfunction matrix at $\mathrm{RX}$ side; and $\mathrm{C}^{T X}$ is the mode intensity matrix at TX side:

$$
\mathbf{E}^{R X}=\left(\begin{array}{cccc}
E_{1,\left(x_{1}, y_{1}\right)}^{\text {eign }} & E_{2,\left(x_{1}, y_{1}\right)}^{\text {eign }} & \cdots & E_{N,\left(x_{1}, y_{1}\right)}^{\text {eign }} \\
E_{1,\left(x_{2}, y_{2}\right)}^{\text {eign }} & E_{2,\left(x_{2}, y_{2}\right)}^{\text {eign }} & \cdots & E_{N,\left(x_{2}, y_{2}\right)}^{\text {eign }} \\
\vdots & \vdots & \ddots & \vdots \\
E_{1,\left(x_{q}, y_{q}\right)}^{\text {eign }} & E_{2,\left(x_{q}, y_{q}\right)}^{\text {eign }} & \cdots & E_{N,\left(x_{q}, y_{q}\right)}^{\text {eign }}
\end{array}\right) ; \quad \mathbf{C}^{T X}=\left(\begin{array}{cccc}
C_{1,\left(x_{1}, y_{1}\right)}^{T X} & C_{1,\left(x_{2}, y_{2}\right)}^{T X} & \cdots & C_{1,\left(x_{p}, y_{p}\right)}^{T X} \\
C_{2,\left(x_{1}, y_{1}\right)}^{T X} & C_{2,\left(x_{2}, y_{2}\right)}^{T X} & \cdots & C_{2,\left(x_{p}, y_{p}\right)}^{T X} \\
\vdots & \vdots & \ddots & \vdots \\
C_{N,\left(x_{1}, y_{1}\right)}^{T X} & C_{N,\left(x_{2}, y_{2}\right)}^{T X} & \cdots & C_{N,\left(x_{p}, y_{p}\right)}^{T X}
\end{array}\right) .
$$

Substitute (11) into (10) and let $G_{t}=G_{r}=1$ for brevity, the MIMO capacity $\mathbb{C}_{\text {MIMO }}$ becomes:

$$
\mathbb{C}_{M I M O}=\log \operatorname{det}\left(\mathbf{I}_{q \times q}+\frac{\rho}{p} \cdot \mathbf{E}^{R X} \cdot \mathbf{D}_{z_{r}}^{M} \cdot \mathbf{C}^{T X} \cdot \mathbf{C}^{T X *} \cdot \mathbf{D}_{z_{r}}^{M *} \cdot \mathbf{E}^{R X *}\right) .
$$

Since $\operatorname{det}(\mathbf{I}+\mathbf{A B})=\operatorname{det}(\mathbf{I}+\mathbf{B A})[28]$, the MIMO capacity becomes:

$$
\mathbb{C}_{M I M O}=\log \operatorname{det}\left[\mathbf{I}_{N \times N}+\frac{\rho}{p}\left(\mathbf{E}^{R X *} \mathbf{E}^{R X}\right) \cdot \mathbf{D}_{z_{r}}^{M}\left(\mathbf{C}^{T X} \mathbf{C}^{T X *}\right) \cdot \mathbf{D}_{z_{r}}^{M *}\right] .
$$

It is difficult to derive the exactly PDF of the MIMO capacity in (13). However, approximate results can be derived if we assume the SNR at the receiver is either high enough or low enough.

1) High SNR Regime Analysis:

Theorem 1: In the high SNR regime, the MIMO capacity in underground tunnels follows a normal distribution.

Proof: As discussed in Section III, only modes that have significant energy need to be considered. Consequently, all the elements on the diagonal of the mode propagation matrix $\mathbf{D}_{z_{r}}^{M}$ is not trivial. Moreocer, in high SNR regime analysis, we assume that the number of TX antenna elements $p$ and RX antenna elements $q$ are larger than the number of significant modes $N$ (i.e. $p \geq N$ and $q \geq N)$. Then $\mathbf{D}_{z_{r}}^{M}, \mathbf{C}^{T X}$ and $\mathbf{E}^{R X}$ are all $N \times N$ full-rank matrix. If the $\mathrm{SNR}$ at the 
RX side is high enough, then the MIMO capacity in (13) can be approximated as

$$
\mathbb{C}_{M I M O}^{\text {high }} \simeq \sum_{l=1}^{N} \log \left(\rho \cdot\left|e^{-\Gamma_{l} \cdot z_{r}} \cdot \prod_{k=1}^{M}\left(1-L_{l}^{k}\right)\right|^{2}\right)+\log \operatorname{det}\left(\mathbf{E}^{R X *} \mathbf{E}^{R X}\right)+\log \operatorname{det}\left(\frac{1}{p} \mathbf{C}^{T X} \mathbf{C}^{T X *}\right) .
$$

The last two terms in (14) are determined by the geometry of the RX and TX antenna elements, respectively. The first term in (14) is the sum of the capacities of $N$ sub-channels. Each subchannel refers to a propagation mode. The capacity of each sub-channel is governed by the tunnel size and the vehicular traffic flow. Since the number, positions, and sizes of the vehicles between the transceivers are random, the capacities of each sub channels are also random. The first term in (14) can be further developed as

$$
\sum_{l=1}^{N} \log \left(\rho \cdot\left|e^{-\Gamma_{l} \cdot z_{r}} \cdot \prod_{k=1}^{M}\left(1-L_{l}^{k}\right)\right|^{2}\right)=\sum_{l=1}^{N}\left(\log \rho+2 \log \left|e^{-\Gamma_{l} \cdot z_{r}}\right|\right)+\sum_{l=1}^{N} \sum_{k=1}^{M} 2 \log \left(1-L_{l}^{k}\right) .
$$

The first sum in (15) is determined by the SNR and the mode attenuation coefficients, which are constants after the communication starts. The second sum in (15) is determined by the traffic flow of random vehicles. The additional loss parameters $\left\{L_{l}^{k}, k=1,2, \ldots M ; l=1,2, \ldots N\right\}$ are independent random variables. Since the tunnel is considered to be long, we assume that the number of vehicles $M$ is correspondingly large. Therefore, the second sum in (15) can be viewed as the sum of a sufficiently large number of independent random variables, each with finite mean and variance. According to the central limit theorem and Lindeberg's condition [29], the second sum in (15) are approximately normally distributed. Then the MIMO capacity in (14) is actually the sum of a normal distributed random variable and several constants. Therefore, the MIMO capacity in high SNR regime follows a normal distribution, which completes the proof.

Since the normal distribution can be completely characterized by its first two moments, we calculate mean and variance of the MIMO capacity in the rest part of this subsection.

Substitute (15) into (14) and calculate the mean value, we derive

$E\left[\mathbb{C}_{\text {MIMO }}^{\text {high }}\right]=\sum_{l=1}^{N}\left(\log \rho+2 \log \left|e^{-\Gamma_{l} \cdot z_{l}}\right|\right)+\log \operatorname{det}\left(\mathbf{E}^{R X *} \mathbf{E}^{R X}\right)+\log \operatorname{det}\left(\frac{1}{p} \mathbf{C}^{T X} \mathbf{C}^{T X *}\right)+E\left[\sum_{l=1}^{N} \sum_{k=1}^{M} 2 \log \left(1-L_{l}^{k}\right)\right]$.

In underground tunnels, vehicular traffic flow can be modeled as a Poisson flow [30]. If the distance between the transceivers is $z_{r}$, the probability that the number of vehicles $M=m$ is:

$$
P(M=m)=\left(\lambda \frac{z_{r}}{v}\right)^{m} \cdot \frac{1}{m !} \cdot e^{-\lambda \frac{z_{r}}{v}},
$$

where $\lambda$ is the average rate of vehicle arrival (vehicles/sec) in the tunnel; and $v$ is the average velocity of the vehicles. Then the last term in (16) can be further developed as

$$
E\left[\sum_{l=1}^{N} \sum_{k=1}^{M} 2 \log \left(1-L_{l}^{k}\right)\right]=\sum_{m=0}^{\infty}\left\{P(M=m) \cdot \sum_{k=1}^{m} E\left[\sum_{l=1}^{N} 2 \log \left(1-L_{l}^{k}\right)\right]\right\} .
$$

Every vehicle runs in one of the $L$ lanes in the tunnel. Hence the x-coordinate of the $i^{\text {th }}$ 
vehicles $x_{v}^{i}$ belongs to $\left\{a\left(\frac{1+2 l}{L}-1\right) \mid l=0,1, \ldots L-1\right\}$. The x-coordinate of the vehicle obeys uniform distribution with the probability $1 / L$. The size of the vehicles are also assumed to have the uniform distribution in the size interval defined in Section III. Then the expectations $E\left[\sum_{l=1}^{N} 2 \log \left(1-L_{l}^{k}\right)\right]$ in (18) are the same for all $k \in\{1,2, \ldots M\}$. Thus, we denote:

$$
\mu_{L}=E\left[\sum_{l=1}^{N} 2 \log \left(1-L_{l}^{k}\right)\right]
$$

which can be calculated using (7). Substituting (17) and (19) into (18) yields

$$
E\left[\sum_{l=1}^{N} \sum_{k=1}^{M} 2 \log \left(1-L_{l}^{k}\right)\right]=\sum_{m=0}^{\infty}\left(\lambda \frac{z_{r}}{v}\right)^{m} \cdot \frac{1}{m !} \cdot e^{-\lambda \frac{z_{r}}{v}} \cdot m \cdot \mu_{L}=\lambda \cdot \frac{z_{r}}{v} \cdot \mu_{L} .
$$

Substituting (20) into (16) yields the mean (ergodic) MIMO capacity in high SNR regime:

$$
E\left[\mathbb{C}_{\text {MIMO }}^{\text {high }}\right]=\sum_{l=1}^{N}\left(\log \rho+2 \log \left|e^{-\Gamma_{l} \cdot z_{r}}\right|\right)+\log \operatorname{det}\left(\mathbf{E}^{R X *} \mathbf{E}^{R X}\right)+\log \operatorname{det}\left(\frac{1}{p} \mathbf{C}^{T X} \mathbf{C}^{T X *}\right)+\lambda \frac{z_{r}}{v} \mu_{L},
$$

The variance of the MIMO capacity in high SNR regime can be calculated in the similar way:

$$
\operatorname{Var}\left[\mathbb{C}_{M I M O}^{h i g h}\right]=\lambda \cdot \frac{z_{r}}{v} \cdot \sigma_{L}^{2}, \quad \text { where } \quad \sigma_{L}^{2}=\operatorname{Var}\left[\sum_{l=1}^{N} 2 \log \left(1-L_{l}^{k}\right)\right],
$$

Note that $\sigma_{L}^{2}$ can also be calculated using (7). After the mean and variance of the MIMO capacity are given, the capacity distribution in high SNR regime is completely characterized.

Corollary 1: In the high SNR regime, the $\epsilon$-outage capacity of the MIMO systems in underground tunnels is given by

$$
\text { Outage }_{\epsilon}\left[\mathbb{C}_{\text {MIMO }}^{h i g h}\right]=E\left[\mathbb{C}_{\text {MIMO }}^{h i g h}\right]+\operatorname{erf}^{-1}(2 \epsilon-1) \cdot \sqrt{2 \operatorname{Var}\left[\mathbb{C}_{\text {MIMO }}^{\text {high }}\right]},
$$

where $\operatorname{erf}^{-1}(x)$ is the inverse function of the error function $\operatorname{erf}(x) ; E\left[\mathbb{C}_{\text {MIMO }}^{\text {high }}\right]$ and $\operatorname{Var}\left[\mathbb{C}_{\text {MIMO }}^{\text {high }}\right]$ are given by (21) and (22), respectively.

Proof: According to Theorem 1, the MIMO capacity in high SNR regime follows the normal distribution $\mathcal{N}\left(E\left[\mathbb{C}_{\text {MIMO }}^{\text {high }}\right], \operatorname{Var}\left[\mathbb{C}_{\text {MIMO }}^{\text {high }}\right]\right)$. Since the $\epsilon$-outage capacity is the capacity guaranteed for $(1-\epsilon)$ of the channel realizations, we have

$$
\epsilon=P_{\mathcal{N}}\left(\mathbb{C}_{\text {MIMO }}^{\text {high }}<\text { Outage }_{\epsilon}\left[\mathbb{C}_{\text {MIMO }}^{\text {high }}\right]\right)=\frac{1}{2}\left[1+\operatorname{erf}\left(\frac{\text { Outage }_{\epsilon}\left[\mathbb{C}_{M I M O}^{\text {high }}\right]-E\left[\mathbb{C}_{\text {MIMO }}^{\text {high }}\right]}{\sqrt{2 \operatorname{Var}\left[\mathbb{C}_{\text {MIMO }}^{\text {high }}\right]}}\right)\right]
$$

Corollary 1 can be derived from (24), which completes the proof.

2) Low SNR Regime Analysis:

Theorem 2: In the low SNR regime, the MIMO capacity in underground tunnels follows a lognormal distribution.

Proof: For brevity, we first use a simple matrix to denote the product of matrixes in (13):

$$
\mathbf{G} \stackrel{\text { def }}{=} \frac{\rho}{p}\left(\mathbf{E}^{R X *} \mathbf{E}^{R X}\right) \cdot \mathbf{D}_{z_{r}}^{M} \cdot\left(\mathbf{C}^{T X} \mathbf{C}^{T X *}\right) \cdot \mathbf{D}_{z_{r}}^{M *}
$$


According to the relationship between matrix determinant and matrix trace [28], (13) can be further developed as

$$
\mathbb{C}_{\text {MIMO }}=\log \operatorname{det}(\mathbf{I}+\mathbf{G})=\log (\exp \{\operatorname{tr}[\log (\mathbf{I}+\mathbf{G})]\})=\log \left\{\sum_{k=0}^{\infty} \frac{1}{k !}\left[-\sum_{j=1}^{\infty} \frac{(-1)^{j}}{j !} \operatorname{tr}\left(\mathbf{G}^{j}\right)\right]^{k}\right\},
$$

where $\operatorname{tr}(\mathbf{X})$ is the trace of the matrix $\mathbf{X}$. In low SNR regime,

$$
|\operatorname{tr}(\mathbf{G})| \gg\left|\frac{(-1)^{j}}{j !} \operatorname{tr}\left(\mathbf{G}^{j}\right)\right|, \quad j>1 ; \quad|\operatorname{tr}(\mathbf{G})| \gg\left|\frac{1}{k !}[-\operatorname{tr}(\mathbf{G})]^{k}\right|, \quad k>1 .
$$

Substituting (27) into (26) yields

$$
\mathbb{C}_{\text {MIMO }}^{\text {low }} \simeq \log [1+\operatorname{tr}(\mathbf{G})] \simeq \operatorname{tr}(\mathbf{G}) \cdot \log e=\frac{\rho}{p} \log e \cdot \sum_{j=1}^{p} \sum_{i=1}^{q}\left|\sum_{l=1}^{N}\left[E_{l,\left(x_{i}, y_{i}\right)}^{e i g n} \cdot C_{l, j}^{T X} \cdot e^{-\Gamma_{l} \cdot z_{r}} \cdot \prod_{k=1}^{M}\left(1-L_{l}^{k}\right)\right]\right|^{2},
$$

For brevity, we denote $f_{l, i, j} \stackrel{\text { def }}{=} E_{l,\left(x_{i}, y_{i}\right)}^{e i g n} \cdot C_{l, j}^{T X} \cdot e^{-\Gamma_{l} \cdot z_{r}}$. Then

$$
\left|\sum_{l=1}^{N}\left[E_{l,\left(x_{i}, y_{i}\right)}^{\text {eign }} C_{l, j}^{T X} \cdot e^{-\Gamma_{l} \cdot z_{r}} \cdot \prod_{k=1}^{M}\left(1-L_{l}^{k}\right)\right]\right|^{2}=\left|\sum_{l=1}^{N}\left\{\left[\operatorname{Re}\left(f_{l, i, j}\right)+j \cdot \operatorname{Im}\left(f_{l, i, j}\right)\right] \cdot \prod_{k=1}^{M}\left(1-L_{l}^{k}\right)\right\}\right|^{2}=X_{1}{ }^{2}+X_{2}{ }^{2},
$$

where

$$
X_{1}=\left|\sum_{l=1}^{N}\left[\operatorname{Re}\left(f_{l, i, j}\right) \cdot \prod_{k=1}^{M}\left(1-L_{l}^{k}\right)\right]\right| ; \quad X_{2}=\left|\sum_{l=1}^{N}\left[\operatorname{Im}\left(f_{l, i, j}\right) \cdot \prod_{k=1}^{M}\left(1-L_{l}^{k}\right)\right]\right| .
$$

where $\operatorname{Re}(x)$ and $\operatorname{Im}(x)$ denote the real and imaginary part of the complex value $x$, respectively;

Again, we assume that the number of vehicles $M$ is large enough so that $\prod_{k=1}^{M}\left(1-L_{l}^{k}\right)$ can be viewed as the product of a sufficiently large number of independent and positive random variables. According to the central limit theorem [29], $\prod_{k=1}^{M}\left(1-L_{l}^{k}\right)$ in (30) approximately follow log-normal distributions. $X_{1}$ and $X_{2}$ shown in (30) are the absolute values of two weighted sums of the log-normal variables $\prod_{k=1}^{M}\left(1-L_{l}^{k}\right)$, respectively. According to [27], it has been well recognized that the sum of log-normal random variables can be well approximated by a new lognormal variable. It can be proved that this result also applies to the absolute values of the weighted sums of log-normal variables. Therefore, $X_{1}$ and $X_{2}$ are also log-normal variables. Moreover, based on the definition of the log-normal distribution, it is easy to prove that the square of a log-normal variable is also a log-normal variable. As a result, $X_{1}{ }^{2}$ and $X_{2}{ }^{2}$ follow the log-normal distribution. The MIMO capacity shown in (26) is the sum of the log-normal variables given in (29). Therefore, the MIMO capacity in low SNR regime follows a log-normal distribution. This completes the proof.

Substitute (29) into (28) and calculate the mean value, we derive

$$
\mathbb{C}_{\text {MIMO }}^{\text {low }}=\frac{\rho}{p} \log e \cdot \sum_{j=1}^{p} \sum_{i=1}^{q}\left(X_{1}^{2}+X_{2}^{2}\right) .
$$

According to the proof of Theorem 2, $X_{1}{ }^{2}$ and $X_{2}{ }^{2}$ are log-normal variables. The mean (or variance) of the sum of the log-normal variable can be approximated calculated by the sum of 
the mean (or variance) of each addend variable [27]. Therefore,

$$
\begin{aligned}
& E\left[\mathbb{C}_{\text {MIMO }}^{\text {low }}\right]=\frac{\rho}{p} \log e \cdot \sum_{j=1}^{p} \sum_{i=1}^{q}\left(E\left[X_{1}^{2}\right]+E\left[X_{2}^{2}\right]\right), \\
& \operatorname{Var}\left[\mathbb{C}_{\text {MIMO }}^{\text {low }}\right] \simeq\left(\frac{\rho}{p} \log e\right)^{2} \cdot \sum_{j=1}^{p} \sum_{i=1}^{q}\left(\operatorname{Var}\left[X_{1}^{2}\right]+\operatorname{Var}\left[X_{2}^{2}\right]\right),
\end{aligned}
$$

In the following, we show the detailed calculating process of $E\left[X_{1}^{2}\right]$ and $\operatorname{Var}\left[X_{1}^{2}\right]$, while $E\left[X_{2}^{2}\right]$ and $\operatorname{Var}\left[X_{2}^{2}\right]$ can be easily derived by exchanging $\operatorname{Re}\left(f_{l, i, j}\right)$ with $\operatorname{Im}\left(f_{l, i, j}\right)$.

According to [26], the mean and variance of the square of a log-normal variable can be expressed as functions of the mean and variance of the log-normal variable. Then we have:

$$
E\left[X_{1}^{2}\right]=E^{2}\left[X_{1}\right]+\operatorname{Var}\left[X_{1}\right], \quad \operatorname{Var}\left[X_{1}^{2}\right]=\left(E^{2}\left[X_{1}\right]+\operatorname{Var}\left[X_{1}\right]\right)^{2}\left[\left(1+\frac{\operatorname{Var}\left[X_{1}\right]}{E^{2}\left[X_{1}\right]}\right)^{4}-1\right],
$$

Next we calculate the mean and variance of lognormal variable $X_{1}$ :

$$
E\left[X_{1}\right]=\left|\sum_{l=1}^{N}\left\{\operatorname{Re}\left(f_{l, i, j}\right) \cdot E\left[\prod_{k=1}^{M}\left(1-L_{l}^{k}\right)\right]\right\}\right|, \quad \operatorname{Var}\left[X_{1}\right] \simeq \sum_{l=1}^{N}\left\{\operatorname{Re}^{2}\left(f_{l, i, j}\right) \cdot \operatorname{Var}\left[\prod_{k=1}^{M}\left(1-L_{l}^{k}\right)\right]\right\},
$$

We first calculate $E\left[X_{1}\right]$ in (35), where

$$
E\left[\prod_{k=1}^{M}\left(1-L_{l}^{k}\right)\right]=\sum_{m=1}^{\infty} P(M=m) \cdot E\left[\prod_{k=1}^{m}\left(1-L_{l}^{k}\right)\right] .
$$

Since the positions and the sizes of the vehicles have independent and identical distributions,

$$
E\left[\prod_{k=1}^{m}\left(1-L_{l}^{k}\right)\right]=\prod_{k=1}^{m} E\left[1-L_{l}^{k}\right]=E^{m}\left[1-L_{l}^{k}\right] \stackrel{\text { def }}{=} \mu_{l}^{m}
$$

where $E\left[1-L_{l}^{k}\right]$ can be calculate using (7). Here we use $\mu_{l}$ to denote $E\left[1-L_{l}^{k}\right]$ for brevity. Substituting (17), (36), and (37) into (35) yields:

$$
E\left[X_{1}\right]=\left|\sum_{l=1}^{N}\left\{\operatorname{Re}\left(f_{l, i, j}\right) \cdot e^{\lambda \frac{z_{r}}{v}\left(\mu_{l}-1\right)}\right\}\right| .
$$

Similarly, we can derive

$$
\operatorname{Var}\left[X_{1}\right] \simeq \sum_{l=1}^{N}\left\{\operatorname{Re}^{2}\left(f_{l, i, j}\right) \cdot\left[e^{\lambda \frac{z_{r}}{v}\left(\mu_{l}^{2}+\sigma_{l}^{2}-1\right)}-e^{\lambda \frac{z_{r}}{v}\left(\mu_{l}^{2}-1\right)}\right]\right\} ; \quad \text { and } \quad \sigma_{l}^{2} \stackrel{\text { def }}{=} \operatorname{Var}\left[1-L_{l}^{k}\right] .
$$

where $\mu_{l}$ is defined in (37); we use $\sigma_{l}^{2}$ to denote $\operatorname{Var}\left[1-L_{l}^{k}\right]$ for brevity.

By substituting (34), (38), (39) into (32) and (33), we can calculate the mean (ergodic capacity) and variance of the MIMO capacity in low SNR regime. Then the outage capacity of the MIMO systems in low SNR regime can be calculated based on the following corollary.

Corollary 2: In the low SNR regime, the $\epsilon$-outage capacity of the MIMO systems in under- 
ground tunnels is given by

$$
\text { Outage }_{\epsilon}\left[\mathbb{C}_{\text {MIMO }}^{\text {low }}\right]=\frac{E\left[\mathbb{C}_{\text {MIMO }}^{\text {low }}\right]}{\sqrt{\kappa}} \cdot e^{\operatorname{erf}^{-1}(2 \epsilon-1) \cdot \sqrt{2 \ln \kappa}}, \text { where } \kappa=1+\frac{\operatorname{Var}\left[\mathbb{C}_{\text {MIMO }}^{\text {low }}\right]}{E^{2}\left[\mathbb{C}_{\text {MIMO }}^{\text {low }}\right]},
$$

where $E\left[\mathbb{C}_{\text {MIMO }}^{\text {low }}\right]$ and $\operatorname{Var}\left[\mathbb{C}_{\text {MIMO }}^{\text {low }}\right]$ are calculated in the above analysis.

Proof: According to Theorem 2, the MIMO capacity in low SNR regime follows the lognormal distribution. Then we have

$$
\epsilon=P_{\log -\mathcal{N}}\left(\mathbb{C}_{\text {MIMO }}^{\text {low }}<\text { Outage }_{\epsilon}\left[\mathbb{C}_{\text {MIMO }}^{\text {low }}\right]\right)=\frac{1}{2}\left[1+\operatorname{erf}\left(\frac{\ln \text { Outage }}{\epsilon}\left[\mathbb{C}_{M I M O}^{\text {low }}\right]-\ln \frac{E\left[\mathbb{C}_{M I M O}^{\text {low }}\right]}{\sqrt{\kappa}}\right)\right],
$$

Corollary 2 can be derived from (41), which completes the proof.

\section{B. MIMO Antenna Geometry Design Scheme}

So far the MIMO capacity in underground tunnels are thoroughly analyzed. In this subsection, the optimal MIMO antenna geometry is designed to maximize the outage capacity. Since the MIMO capacity in tunnels have different attributes in high SNR and low SNR regime, in this subsection, we first develop the optimal MIMO antenna geometry in high SNR regimes. Then this optimal MIMO antenna geometry is modified to fit the attributes in the low SNR regime.

1) MIMO Antenna Geometry in High SNR Regime: In (23), the high SNR $\epsilon$-outage capacity is determined by the mean and variance of the high SNR MIMO capacity. According to (21) and (22), the TX and RX antenna geometries can only affect the mean capacity in (21) but have no effect on the capacity variance in (22). As a result, in high SNR regime, to maximize the $\epsilon$-outage capacity is equal to maximize the mean capacity. Hence, in the next step, we design the TX and RX antenna geometry to maximize the mean capacity given in (21).

The first term and the last term in (21) are determined by the tunnel size and the vehicular traffic flow between the transceivers, which are not affected by the TX and RX antenna geometry. The optimal antenna geometries are expected to maximize the second and third term in (21). It is equal to maximize $\operatorname{det}\left(\mathbf{E}^{R X *} \mathbf{E}^{R X}\right)$ by selecting optimal $\mathrm{RX}$ antenna geometry, and to maximize $\operatorname{det}\left(\frac{1}{p} \mathbf{C}^{T X} \mathbf{C}^{T X *}\right)$ by selecting optimal TX antenna geometry.

According to (1), the eigenfunctions of different modes are orthogonal to each other:

$$
\int_{-a}^{a} \int_{-b}^{b} E_{i,(x, y)}^{e i g n} \cdot E_{j,(x, y)}^{e i g n *} d x d y \simeq \begin{cases}a b, & \text { if } i=j \\ 0, & \text { otherwise }\end{cases}
$$

At $\mathrm{RX}$ side, the matrix $\mathbf{E}^{R X *} \mathbf{E}^{R X}$ is in fact the covariance matrix of the eigenfunctions of all significant modes at the positions of the RX antenna elements. Due to the mode orthogonality, $\operatorname{det}\left(\mathbf{E}^{R X *} \mathbf{E}^{R X}\right)$ is maximized if $\mathbf{E}^{R X *} \mathbf{E}^{R X}$ is diagonal. If the $\mathrm{RX}$ antenna elements are placed at all the positions where the eigenfunctions of significant modes have extrema values, the matrix 
$\mathbf{E}^{R X} \cdot \mathbf{E}^{R X *}$ can be approximately diagonalized. The maximum value of $\operatorname{det}\left(\mathbf{E}^{R X *} \mathbf{E}^{R X}\right)$ is

$$
\max \left\{\operatorname{det}\left(\mathbf{E}^{R X *} \mathbf{E}^{R X}\right)\right\} \simeq \prod_{i=1}^{N}\left[\sum_{i=1}^{q}\left|E_{i,(x, y)}^{\text {eign }}\right|^{2}\right] \simeq q^{N} .
$$

At TX side, we first check the mode intensity $C_{m n, j}^{T X}$ given in (3). Since $\sqrt{1-\left(\frac{m \pi}{2 a k}\right)^{2}-\left(\frac{n \pi}{2 b k}\right)^{2}} \simeq 1$ for low order modes (i.e. the significant modes considered in this paper), $C_{m n, j}^{T X}$ approximately equals to the mode eigenfunction multiplied by a constant. Hence, the matrix $\mathbf{C}^{T X} \mathbf{C}^{T X *}$ can also be viewed as the covariance matrix of the eigenfunctions of all significant modes at the positions of the TX antenna elements. Therefore, in high SNR regime, the optimal TX antenna shares the same geometry as the optimal RX antenna geometry, where antenna elements are placed at all the positions where the eigenfunctions of significant modes have extrema values. And

$$
\max \left\{\operatorname{det}\left(\frac{1}{p} \mathbf{C}^{T X} \mathbf{C}^{T X *}\right)\right\} \simeq \prod_{i=1}^{N}\left[\sum_{j=1}^{p} \frac{1}{p}\left|\frac{\pi}{a b} E_{j,(x, y)}^{e i g n}\right|^{2}\right] \simeq\left(\frac{\pi}{a b}\right)^{2 N}
$$

Based on the above discussion, the optimal RX and TX MIMO antenna geometry in high SNR regime is described as follows: the number and geometry of the antenna elements depend on which modes have significant power in the tunnel. For mode $E M_{m n}, p=q=m \times n$ antenna elements are needed. Their positions $\left\{\left(x_{u}, y_{v}\right)\right\}$ should be

$$
x_{u}=-a+\left(u-\frac{1}{2}\right) \frac{2 a}{m}, \quad u \in[1, m] ; \quad y_{v}=-b+\left(v-\frac{1}{2}\right) \frac{2 b}{n}, \quad v \in[1, n]
$$

Since lower order modes have lower attenuation rates, the probability that the lower order modes have significant power is higher. Therefore, lower order modes are first to be considered when the number of available antenna elements is limited. For example, if we only have one antenna element, the lowest order mode $E H_{11}$ is considered. The position of the antenna element is $(0,0)$. If we have three antenna elements, both mode $E H_{11}$ and $E H_{21}$ are considered. The positions of the three antenna elements are $(0,0),(-a / 2,0)$ and $(a / 2,0)$, so on and so forth.

Intuitively, in high SNR regime, each significant mode needs a set of TX and RX antenna elements to be efficiently excited and received. By substituting (43) and (44) into (21) and (23), we find that the high SNR $\epsilon$-outage capacity increases linearly with $\log q$ where $q$ is the number of RX elements. It indicates that more RX elements are always favorable although the capacity increase becomes trivial when $q$ is larger than a threshold. In contrast, the high SNR $\epsilon$-outage capacity remains the same as the number of TX elements increases, which is because that the total TX power $P_{t o t}$ is a constant and is equally divided and allocated to each TX elements.

2) MIMO Antenna Geometry in Low SNR Regime: In low SNR regime, the optimal RX antenna geometry designed for high SNR regime still works, since the proposed RX antenna geometry can effectively receive all significant propagation modes no matter whether the SNR is high or low. However, the number and geometry of the TX antenna elements in low SNR regime need to be redesigned since it may be not efficient to involve a large number of TX 
antenna elements to excite all propagation modes in low SNR regime.

Based on the above discussion, our goal in this subsection is to maximize the low SNR $\epsilon$ outage capacity in (40) by designing the number and geometry of the TX antenna elements while keep the RX antenna geometry designed for high SNR regime. In (40), the low SNR $\epsilon$-outage capacity is determined by the mean capacity $E\left[\mathbb{C}_{\text {MIMO }}^{\text {low }}\right]$ and the coefficient $\kappa$, where $\kappa$ is determined by the ratio $\frac{\operatorname{Var}\left[\mathbb{C}_{M I M O}^{\text {low }}\right]}{E^{2}\left[\mathbb{C}_{M I M O}^{\text {low }}\right]}$, which can be approximately viewed as a constant if only antenna geometry can be changed, according to (32) - (39). Hence, the coefficient $\kappa$ becomes a constant. As a result, in low SNR regime, to maximize the $\epsilon$-outage capacity is equal to maximize the mean capacity $E\left[\mathbb{C}_{M I M O}^{l o w}\right]$, in the condition that only the antenna geometry can be designed.

Since the optimal RX antenna geometry for high SNR regime is also utilized in low SNR regime, the correlation matrix $\mathbf{E}^{R X *} \mathbf{E}^{R X}$ becomes a diagonal matrix. Then (25) and (28) becomes:

$$
\begin{aligned}
\mathbb{C}_{\text {MIMO }}^{l o w} & \simeq \log e \cdot \operatorname{tr}\left[\frac{\rho}{p}\left(\mathbf{E}^{R X *} \mathbf{E}^{R X}\right) \cdot \mathbf{D}_{z_{r}}^{M} \cdot\left(\mathbf{C}^{T X} \mathbf{C}^{T X *}\right) \cdot \mathbf{D}_{z_{r}}^{M *}\right]=\log e \cdot \operatorname{tr}\left[\frac{\rho}{p} \cdot q \cdot \mathbf{I} \cdot \mathbf{D}_{z_{r}}^{M} \cdot\left(\mathbf{C}^{T X} \mathbf{C}^{T X *}\right) \cdot \mathbf{D}_{z_{r}}^{M *}\right] \\
& =\log e \cdot \operatorname{tr}\left[\frac{q \rho}{p}\left(\mathbf{C}^{T X} \mathbf{C}^{T X *}\right) \cdot\left(\mathbf{D}_{z_{r}}^{M *} \mathbf{D}_{z_{r}}^{M}\right)\right]=\frac{q \rho}{p} \log e \cdot \sum_{l=1}^{N} \sum_{j=1}^{p}\left|C_{l, j}^{T X}\right|^{2} \cdot\left|e^{-\Gamma_{l} \cdot z_{r}} \cdot \prod_{k=1}^{M}\left(1-L_{l}^{k}\right)\right|^{2} \cdot \quad \text { (46) }
\end{aligned}
$$

To determine the optimal number and positions of the TX antenna elements in low SNR regime, we first assume that a sufficient large number of TX antenna elements are placed at the whole tunnel cross section. Then we define a set of indicators $\left\{\delta_{j}, j=1,2, \ldots, p\right\}$ where $\delta_{j}=1$ if the $j^{\text {th }} \mathrm{TX}$ antenna element is actually used, otherwise $\delta_{j}=0$. Then (46) becomes:

$$
\mathbb{C}_{\text {MIMO }}^{\text {low }} \simeq \frac{q \rho \log e}{\sum_{j=1}^{p} \delta_{j}} \cdot \sum_{j=1}^{p} \delta_{j} \cdot \sum_{l=1}^{N}\left|C_{l, j}^{T X} \cdot e^{-\Gamma_{l} \cdot z_{r}}\right|^{2} \cdot \prod_{k=1}^{M}\left(1-L_{l}^{k}\right)^{2} .
$$

Hence,

$$
\begin{aligned}
& E\left[\mathbb{C}_{M I M O}^{l o w}\right] \\
& \simeq \frac{q \rho \log e}{\sum_{j=1}^{p} \delta_{j}} \cdot \sum_{j=1}^{p} \delta_{j} \cdot \sum_{l=1}^{N}\left|C_{l, j}^{T X} \cdot e^{-\Gamma_{l} \cdot z_{r}}\right|^{2} \cdot E\left[\prod_{k=1}^{M}\left(1-L_{l}^{k}\right)^{2}\right]=\frac{q \rho \log e}{\sum_{j=1}^{p} \delta_{j}} \cdot \sum_{j=1}^{p} \delta_{j} \cdot \sum_{l=1}^{N}\left|C_{l, j}^{T X} \cdot e^{-\Gamma_{l} \cdot z_{r}}\right|^{2} \cdot e^{\lambda \frac{z_{r}}{v}\left(\mu_{l}^{2}+\sigma_{l}^{2}-1\right)},
\end{aligned}
$$

where $\mu_{l}$ and $\sigma_{l}^{2}$ is defined in (37) and (39), respectively. Therefore, to find the optimal TX antenna geometry in low SNR regime, it is equal to an optimization problem, which is:

$$
\begin{aligned}
\text { Find }: & \left\{\delta_{j}, j=1,2, \ldots, p\right\} \\
\text { Maximize }: & \frac{q \rho \log e}{\sum_{j=1}^{p} \delta_{j}} \cdot \sum_{j=1}^{p} \delta_{j} \cdot \sum_{l=1}^{N}\left|C_{l, j}^{T X} \cdot e^{-\Gamma_{l} \cdot z_{r}}\right|^{2} \cdot e^{\lambda \frac{z_{r}}{v}\left(\mu_{l}^{2}+\sigma_{l}^{2}-1\right)}
\end{aligned}
$$

It is not difficult to find the solution of this optimization problem, which is: $\delta_{k}=1$ if $k=$ $\arg \max _{j} \sum_{l=1}^{N}\left|C_{l, j}^{T X} \cdot e^{-\Gamma_{l} \cdot z_{r}}\right|^{2} \cdot e^{\lambda \frac{z_{r}}{v}\left(\mu_{l}^{2}+\sigma_{l}^{2}-1\right)}$; otherwise $\delta_{k}=0$. Therefore, the optimal TX antenna geometry in low SNR regime involves only one antenna element. According to the channel model given in Section III, the position of the optimal TX antenna element is located at the 
center of the tunnel cross section, i.e., $(x, y)=(0,0)$.

To sum up, the optimal RX antenna geometry in low SNR regime is the same as in the high SNR regime. The optimal TX antenna geometry in low SNR regime involves only one antenna element that is located at the center of the tunnel cross section. This results can be intuitively explained as follows. In low SNR regime, given a fixed total transmitting power $P_{t o t}$, it is more efficient to concentrate all TX power at the antenna element that can achieves smallest path loss.

\section{CAPacity and Outage Behavior of Cooperative Communication Systems in \\ UNDERGROUND TUNNELS}

In many applications, such as wireless sensor networks, it is impossible to place multiple antenna elements on a single device due to the limited size and cost. In this case, instead of MIMO, cooperative communication systems can be utilized. Based on the channel model in Section III and the MIMO capacity analysis in Section IV, the capacity and outage behavior of the cooperative communication systems in underground tunnels are investigated in this section.

\section{A. Capacity and Outage Probability}

In this subsection, we investigate the capacity and the outage performance of the selection Decode-and-Forward (DF) cooperative scheme [20], which has been widely recognized. The selection DF scheme consists of two phases. In the first phase, the source node sends out the information, which is received by both the relay node and the destination node. In the second phase, the relay node check the SNR of the received signal. If the SNR is above a threshold, the relay node decodes and forwards the received data to the destination node. Otherwise, the source nodes just send the information again to the destination node. According to [20], the channel capacity of this cooperative system is given by

$$
\mathbb{C}_{\text {coop }}= \begin{cases}\frac{1}{2} \log \left(1+2 \rho\left|h_{s d}\right|^{2}\right), & \text { if }\left|h_{s r}\right|^{2}<\frac{e R-1}{\rho} \\ \frac{1}{2} \log \left(1+\rho\left|h_{s d}\right|^{2}+\rho\left|h_{r d}\right|^{2}\right), & \text { if }\left|h_{s r}\right|^{2} \geq \frac{e R-1}{\rho}\end{cases}
$$

where $h_{s d}, h_{r d}$, and $h_{s r}$ are the channel gains between the source and the destination, the relay and the destination, and the source and the relay, respectively; $\rho$ is the SNR at the transmitter; $R$ is the expected data rate. In underground tunnels, the channel gain $h_{s d}, h_{r d}$, and $h_{s r}$ can be calculated by (8). Then the instantaneous cooperative capacity in underground tunnels can be derived. However, the distribution of the cooperative capacity in underground tunnels does not fall into any type of classical random distributions. In the following, we calculate the outage

probability to characterize this distribution. The outage probability of the DF cooperative scheme 
can be derived according to (49), which is given by:

$$
P\left[\mathbb{C}_{\text {coop }}<R\right]=P\left(\left|h_{s r}\right|^{2}<\frac{e^{R}-1}{\rho}\right) \cdot P\left(\left|h_{s d}\right|^{2}<\frac{e^{R}-1}{2 \rho}\right)+P\left(\left|h_{s r}\right|^{2} \geq \frac{e^{R}-1}{\rho}\right) \cdot P\left(\left|h_{s d}\right|^{2}+\left|h_{r d}\right|^{2}<\frac{e^{R}-1}{\rho}\right) .
$$

According to (50), we find that the outage probability is determined by the channel gains $\left|h_{s d}\right|^{2}$, $\left|h_{r d}\right|^{2}$, and $\left|h_{s r}\right|^{2}$. In Section 4.1.2, the square of the channel gain norm $\left|h_{i j}\right|^{2}$ is developed as (29), which is proved to follow a log-normal distribution. The mean and variance of this log-normal variable, $E\left[\left|h_{i j}\right|^{2}\right]$ and $\operatorname{Var}\left[\left|h_{i j}\right|^{2}\right]$, can be calculated using (34)-(39). Then,

$$
P\left(\left|h_{i j}\right|^{2}<\frac{e^{R}-1}{\rho}\right)=\frac{1}{2}\left[1+\operatorname{erf}\left(\frac{\ln \frac{e R-1}{\rho}-\mu_{\left|h_{i j}\right|^{2}}}{\sqrt{2} \cdot \sigma_{\left|h_{i j}\right|^{2}}}\right)\right],
$$

where

$$
\mu_{\left|h_{i j}\right|^{2}}=\ln \left(E\left[\left|h_{i j}\right|^{2}\right]\right)-\frac{1}{2} \ln \left(1+\frac{\operatorname{Var}\left[\left|h_{i j}\right|^{2}\right]}{E^{2}\left[\left|h_{i j}\right|^{2}\right]}\right) ; \quad \sigma_{\left|h_{i j}\right|^{2}}^{2}=\ln \left(1+\frac{\operatorname{Var}\left[\left|h_{i j}\right|^{2}\right]}{E^{2}\left[\left|h_{i j}\right|^{2}\right]}\right) .
$$

The sum of two log-normal variable, $\left|h_{s d}\right|^{2}+\left|h_{r d}\right|^{2}$, also follows a log-normal distribution:

$$
P\left(\left|h_{s d}\right|^{2}+\left|h_{r d}\right|^{2}<\frac{e^{R}-1}{\rho}\right)=\frac{1}{2}\left[1+\operatorname{erf}\left(\frac{\ln \frac{e R-1}{\rho}-\mu_{\left(\left|h_{s d}\right|^{2}+\left|h_{r d}\right|^{2}\right)}}{\sqrt{2} \cdot \sigma_{\left(\left|h_{s d}\right|^{2}+\left|h_{r d}\right|^{2}\right)}}\right)\right],
$$

where

$$
\begin{aligned}
& \mu_{\left(\left|h_{s d}\right|^{2}+\left|h_{r d}\right|^{2}\right)}=\ln \left(E\left[\left|h_{s d}\right|^{2}\right]+E\left[\left|h_{r d}\right|^{2}\right]\right)-\frac{1}{2} \ln \left(1+\frac{\operatorname{Var}\left[\left|h_{s d}\right|^{2}\right]+\operatorname{Var}\left[\left|h_{r d}\right|^{2}\right]}{\left(E\left[\left|h_{s d}\right|^{2}\right]+E\left[\left|h_{r d}\right|^{2}\right]\right)^{2}}\right) ; \\
& \sigma_{\left(\left|h_{s d}\right|^{2}+\left|h_{r d}\right|^{2}\right)}^{2}=\ln \left(1+\frac{\operatorname{Var}\left[\left|h_{s d}\right|^{2}\right]+\operatorname{Var}\left[\left|h_{r d}\right|^{2}\right]}{\left(E\left[\left|h_{s d}\right|^{2}\right]+E\left[\left|h_{r d}\right|^{2}\right]\right)^{2}}\right) .
\end{aligned}
$$

Substituting (51) and (53) into (50) yields the outage probability in underground tunnels:

$$
\begin{aligned}
P\left[\mathbb{C}_{\text {coop }}<R\right]=\frac{1}{4}[1+\operatorname{erf} & \left.\left(\frac{\ln \frac{e R-1}{\rho}-\mu_{\left|h_{s r}\right|^{2}}}{\sqrt{2} \cdot \sigma_{\left|h_{s r}\right|^{2}}}\right)\right] \cdot\left[1+\operatorname{erf}\left(\frac{\ln \frac{e R-1}{2 \rho}-\mu_{\left|h_{s d}\right|^{2}}}{\sqrt{2} \cdot \sigma_{\left|h_{s d}\right|^{2}}}\right)\right] \\
+ & \frac{1}{4}\left[1-\operatorname{erf}\left(\frac{\ln \frac{e R-1}{\rho}-\mu_{\left|h_{s r}\right|^{2}}}{\sqrt{2} \cdot \sigma_{\left|h_{s r}\right|^{2}}}\right)\right] \cdot\left[1+\operatorname{erf}\left(\frac{\ln \frac{e R-1}{\rho}-\mu_{\left(\left|h_{s d}\right|^{2}+\left|h_{r d}\right|^{2}\right)}}{\sqrt{2} \cdot \sigma_{\left(\left|h_{s d}\right|^{2}+\left|h_{r d}\right|^{2}\right)}}\right)\right] .
\end{aligned}
$$

Note that the $\epsilon$-outage cooperative capacity Outage $\epsilon\left[\mathbb{C}_{\text {coop }}\right]$ can be also calculated from (55) by letting $R=$ Outage $_{\epsilon}\left[\mathbb{C}_{\text {coop }}\right]$ and $P\left[\mathbb{C}_{\text {coop }}<\right.$ Outage $\left._{\epsilon}\left[\mathbb{C}_{\text {coop }}\right]\right]=\epsilon$.

\section{B. Optimal Relay Assignment in Tunnels}

So far we assume that the relay position is determined. However, in real applications, especially the mobile wireless networks, the positions of the cooperative users are highly dynamic. Since the relay position has obvious influence on the outage probability, the cooperative relay assignment is of great important. In this subsection, we first use the outage probability given in (55) as the relay assignment metric and propose an outage-optimal and fully distributed cooperative relay assignment protocol. Then, we develop a much simpler relay assignment metric based on the outage probability to reduce the computation burden on each cooperative node. 
1) Outage-Optimal Relay Assignment Protocol: Assuming that in an underground tunnel, the source node $s$ is to send data to the destination node $d$. Node $s$ assign one of its neighbors $\left\{r_{i}, i=1,2, \ldots\right\}$ as the relay node and adopts the selection DF scheme. All the nodes have its own position information, which is valid in most applications. The source first sends RTS message to all its neighbors. RTS message contains the position information of the source and the destination. Each neighbor that can correctly receives the RTS message calculates the relay assignment metric. Here we first use the outage probability given in (55) as the relay assignment metric. To calculate this metric, the following information is required: 1) the position information of itself, the source, and the destination, and 2) the statistical vehicular traffic load information in the underground tunnel, which is assume to be available at each node. No information exchanging among neighbor nodes and the destination node is required.

After deriving the relay assignment metric, each neighbor node randomly select a back-off time and start timing. The mean value of the back-off time is proportional to the relay assignment metric, i.e. the outage probability. The neighbor node sends out a CTS message to the source node after the back-off time out if it does not receive any other CTS messages from other neighbors during the back-off time. Then the source node assigns this neighbor as the relay node and start the selection decode-and-forward cooperative communication. By this relay assignment protocol, the neighbor node that has the least outage probability will be selected as the cooperative relay. Therefore, the protocol is outage-optimal. Moreover, since calculating the relay assignment metric only require the the source and destination position information in the RTS message and the position information of each neighbor node itself, the proposed protocol is also fully distributed.

2) Simpler Relay Assignment Metric: Despite the advantages of the proposed relay assignment protocol, the computation burden to calculate the outage probability in (55) may be heavy for low cost and resource limited devices, such as the sensor nodes. Therefore, we propose a simpler relay assignment metric based on the outage probability given in (55).

According to (55), to minimize the outage probability is equal to maximize the parameters $\mu_{\left|h_{s r}\right|^{2}}$ and $\mu_{\left(\left|h_{s i l}\right|^{2}+\left|h_{r r \mid}\right|^{2}\right)}$ while minimizing the parameters $\sigma_{\left|h_{s r}\right|^{2}}$ and $\sigma_{\left(\left|h_{s l}\right|^{2}+\left|h_{r l}\right|^{2}\right)}$. Similar to the analysis in Section 4.2.2, it can be proved that the position of the relay node does not significantly affect the ratio $\frac{\operatorname{Var}\left[\left|h_{s d}\right|^{2}\right]+\operatorname{Var}\left[\left|h_{r d}\right|^{2}\right]}{\left(E\left[\left|h_{s d}\right|^{2}\right]+E\left[\left|h_{r d}\right|^{2}\right]\right)^{2}}$ and the ratio $\frac{\operatorname{Var}\left[\left|h_{s r}\right|^{2}\right]}{E^{2}\left[\left|h_{s r}\right|^{2}\right]}$. Hence, according to (52) and (54), to minimize the outage probability in (55) is equal to maximize $\ln \left(E\left[\left|h_{s r}\right|^{2}\right]\right)$ and $\ln \left(E\left[\left|h_{s d}\right|^{2}\right]+E\left[\left|h_{r d}\right|^{2}\right]\right)$. Since $E\left[\left|h_{s d}\right|^{2}\right]$ is determined, ony $E\left[\left|h_{s r}\right|^{2}\right]$ and $E\left[\left|h_{r d}\right|^{2}\right]$ need to be considered. However, there exists tradeoff between $\left|h_{s r}\right|$ and $\left|h_{r d}\right|$. The optimal relay node should be placed at the position where $\left|h_{s r}\right|$ and $\left|h_{r d}\right|$ are both sufficient large. Motivated by the above discussion, to reduce the metric computation complexity, we use $\min \left\{E\left[\left|h_{s r}\right|\right], E\left[\left|h_{r d}\right|\right]\right\}$ as a simpler metric instead of the outage probability.

This simpler metric metric can be calculated based on (29), (30), and (38), which can be further simplified by the following approximation. Since the lowest order mode $E H_{11}$ has the 
lowest attenuation rate in tunnels, if the distance between source and destination is large enough, we can approximately assume that only the lowest order mode exists. This assumption is valid since the cooperative communication is not necessary in short range distance communication where the impact of multipath fading is negligible. Then the simpler metric can be calculated as

$$
\begin{aligned}
\min \left\{E\left[\left|h_{s r}\right|\right], E\left[\left|h_{r d}\right|\right]\right\} & \\
& \simeq \min \left\{\left|\frac{\pi}{a b} E_{1,\left(x_{r}, y_{r}\right)}^{\text {eign }} \cdot E_{1,\left(x_{s}, y_{s}\right)}^{e i g n} \cdot e^{z_{s r} \cdot\left[-\Gamma_{1}+\frac{\lambda}{v}\left(\mu_{1}-1\right)\right]}\right|,\left|\frac{\pi}{a b} E_{1,\left(x_{r}, y_{r}\right)}^{\text {eign }} \cdot E_{1,\left(x_{d}, y_{d}\right)}^{e i g n} \cdot e^{z_{r d} \cdot\left[-\Gamma_{1}+\frac{\lambda}{v}\left(\mu_{1}-1\right)\right]}\right|\right\},
\end{aligned}
$$

where $z_{s r}$ is the axial distance between the source and the relay; $z_{r d}$ is the axial distance between the destination and the relay; $\Gamma_{1}$ is the attenuation coefficient of mode $E H_{11} ; E_{1,(x, y)}^{\text {eign }}$ is given in $(1) ; \mu_{1}$ is defined in (37).

The simpler relay assignment metric given in (56) can be adopted in resource limited devices instead of the outage probability given in (55). Note that the back-off time is inversely proportional to this simpler relay assignment metric.

\section{NumeriCAL ANALYSIS}

In this section, the capacity and outage behavior of MIMO and cooperative communication systems in underground tunnels are numerically analyzed. Then, the proposed MIMO antenna geometry as well as the cooperative relay assignment protocol are compared with existing geometry and protocols, respectively. Except studying the effect of certain parameters, the default simulation parameters are set as follows: The one-lane tunnel has a rectangle cross section with a height of $4 \mathrm{~m}$ and a width of $6 \mathrm{~m}$. The operating frequency is $1 \mathrm{GHz}$. The SNR at the transmitter

$\rho=\frac{P_{t o t}}{N_{0}}$ is $100 \mathrm{~dB}$. The traffic load is described using the average rate of vehicle arrival $\lambda$ and average vehicle velocity $v$. The default values are set as $\lambda=0.5 \mathrm{~s}^{-1}$ and $v=72 \mathrm{~km} / \mathrm{hour}$.

\section{A. MIMO Systems in Underground Tunnels}

Fig. 2 shows the ergodic and the 10\%-outage MIMO capacity as functions of of the axial distance between the transceivers, where the $5 \times 5$ MIMO antenna with optimal geometry is adopted. It shows that the theoretical results in high SNR regime have a good match with the simulations when the axial distance is smaller than $500 \mathrm{~m}$, while the theoretical results in low SNR regime have a good match with the simulations when the axial distance is larger than $1000 \mathrm{~m}$. Therefore, the theoretical ergodic and outage MIMO capacities have a good match with the simulations in both high SNR regime and low SNR regime.

In Fig. 3(a), the cumulative distribution function (CDF) of the normal distribution with parameters derived in high SNR regime is compared with the simulated capacity distribution when axial distance is $400 \mathrm{~m}$. In Fig. 3(b), the CDF of the log-normal distribution with parameters derived in low SNR regime is compared with the simulated capacity distribution when axial distance is $1200 \mathrm{~m}$. A good match is shown between the theoretical and simulation results. 
Fig. 4(a) and Fig. 4(b) show the 10\%-Outage capacity of the optimal MIMO antenna geometries with different element number as a function of the axial distance and the SNR at the transmitter, respectively. The capacity of a traditional linear antenna array with 3 antenna elements is also provided for comparison. For fair comparison, one terminal of the traditional linear antenna array is placed at the center of the tunnel. The array is placed parallel to the floor with interval of one wavelength $(0.33 \mathrm{~m})$. Fig. 4(a) and Fig. 4(b) indicate that, the MIMO capacity with optimal geometry is significantly higher than that of the undesigned geometry. In low SNR regime, the undesigned linear array has even smaller capacity than that of the SISO $(1 \times 1)$ system placed at the optimal position. In high SNR regime (axial distance smaller than $500 \mathrm{~m}$ ), the optimal $3 \times 3 \mathrm{MIMO}$ antenna achieves much higher capacity than the optimal $1 \times 3$ SIMO antenna. In low SNR regime (axial distance larger than $600 \mathrm{~m}$ ), the optimal $1 \times 3 \mathrm{SIMO}$ antenna achieves higher outage capacity than the optimal $3 \times 3$ MIMO antenna. This phenomenon is consistent with our analysis on the MIMO antenna geometry design in Section IV-B, i.e. the spatial diversity at the TX side is more efficient in the high SNR regime that in the low SNR regime.

Moreover, Fig. 4(a) and Fig. 4(b) also show the capacity of a $3 \times 3$ MIMO system and a SISO system in terrestrial environments. A widely used terrestrial channel model is utilized, where the Friis transmission equation with a exponent of 3 is used to model the path loss and a Rayleigh random variable is used to model the multipath fading. The MIMO antenna elements are placed far enough to guarantee they are not correlated. Fig. 4(a) and Fig. 4(b) show that the capacity of MIMO and SISO system is much smaller than the capacity in tunnels since the signal energy in tunnels does not spread as much as in terrestrial environments. Meanwhile, the gain of MIMO compared with SISO in terrestrial environments is larger than in tunnels since the MIMO antenna elements in tunnels are more correlated.

\section{B. Cooperative Communication Systems in Underground Tunnels}

In the following analysis on cooperative communication systems in tunnels, we assume the source node and the destination node are $400 \mathrm{~m}$ apart. Both nodes are placed at the center of the tunnel cross section. First, we assume that the relay node is fixed, which is placed in the middle point of the distance between the source and the destination, i.e., at the center of the tunnel cross section and $200 \mathrm{~m}$ apart from both the source and the destination. In Fig. 5, it is shown that the theoretical outage probability is consistent with the simulation results with different traffic load (average arrival rate $\lambda$ ). As the traffic load become heavier, the mean cooperative capacity decreases and the variance of the cooperative capacity increases, which is due to the reason that heavier traffic load causes higher additional path loss and more randomness.

Then, the performance of the relay assignment protocols is analyzed. In the following simulations, we let 50 cooperative relay nodes uniformly distributed between the source and the 
destination. The position of each relay node on the tunnel cross section is also uniformly distributed. Four relay assignment strategies are adopted: 1) optimal strategy that use the outage probability given in (55) as the metric; 2) simpler metric strategy that use the metric given in $(56) ; 3)$ nearest neighbor strategy given in [24] that select the nearest neighbor as the relay node; and 4) farthest neighbor strategy that that select the farthest neighbor as the relay node. In Fig. 6, the outage probability of the four strategies are provided as functions of the TX SNR and the traffic load $(\lambda)$. As expected, the optimal strategy achieves the lowest outage probability in all conditions. The simpler metric strategy has higher outage probability than the optimal strategy but much lower than the nearest and farthest neighbor strategy.

\section{CONCLUSION}

In this paper, we analyze the capacity and outage behavior of the MIMO and cooperative communication systems based on the underground tunnel channel model. For MIMO system, we prove that the MIMO capacity in underground tunnels follows either a normal distribution in high SNR regime or a log-normal distribution in low SNR regime. The ergodic and outage capacity of MIMO systems in tunnels are explicitly expressed as functions of tunnel size, transmission power, vehicular traffic load, and MIMO antenna geometry. Then the optimal MIMO antenna geometry design scheme is proposed, which obviously increases the outage capacity. For cooperative communication systems, we calculate the outage probability of such systems in underground tunnels. Based on the formula of the outage probability, we proposed an outageoptimal cooperative relay assignment protocol, which significantly outperforms the existing relay assignment protocols in term of outage probability in underground tunnel environments.

\section{ACKNOWLEDGMENT}

This work was supported by the US National Science Foundation (CCF-0728889).

\section{REFERENCES}

[1] I. F. Akyildiz, Z. Sun and M. C. Vuran, "Signal Propagation Techniques for Wireless Underground Communication Networks," Physical Communication Journal (Elsevier), Vol. 2, No. 3, pp.167-183, September 2009.

[2] Z. Sun and I. F. Akyildiz, "Channel Modeling of Wireless Networks in Tunnels," in Proc. IEEE GLOBECOM 2008, New Orleans, USA, November 2008.

[3] Z. Sun and I. F. Akyildiz, "Channel Modeling and Analysis for Wireless Networks in Underground Mines and Road Tunnels,” IEEE Trans. Communications, Vol. 58, No. 6, pp. 1758-1768, June 2010.

[4] Z. Sun and I. F. Akyildiz, "Influences of Vehicles on Signal Propagation in Road Tunnels," in Proc. IEEE ICC 2010, Cape Town, South Africa, May 2010.

[5] Z. Sun and I. F. Akyildiz, "A Mode-Based Approach for Channel Modeling in Underground Tunnels under the Impact of Vehicular Traffic Flow," submitted to IEEE Trans. Wireless Communications, in April 2010, revised in March 2011 and June 2011.

[6] D. G. Dudley, M. Lienard, S. F. Mahmoud, and P. Degauque, "Wireless Propagation in Tunnels," IEEE Antenna and Propagation Magazine, vol. 49, no. 2, pp. 11-26, April 2007. 
[7] S. F. Mahmoud and J. R. Wait, "Geometrical Optical approach for Electromagnetic Wave Propagation in Rectangular Mine Tunnels," Radio Science, vol. 9, no. 12, pp. 1147-1158, December 1974.

[8] Y. Hwang, Y. P. Zhang, and R. G. Kouyoumjian, "Ray-Optical Prediction of Radio-Wave Propagation Characteristics in Tunnel Environments Part 1: Theory and Part 2: Analysis and Measurements" IEEE Trans. Antenna and Propagation, vol. 46, no. 9, pp. 1328-1345, September 1998.

[9] A. G. Emslie, R. L. Lagace, and P. F. Strong, "Theory of the Propagation of UHF Radio Waves in Coal Mine Tunnels," IEEE Trans. on Antenna and Propagation, vol. AP-23, no. 2, pp. 192-205, March 1975.

[10] K. D. Laakmann and W. H. Steier, "Waveguides: Characteristic Modes of Hollow Rectangular Dielectric Waveguides", Applied Optics, vol. 15, no. 5, pp. 1334-1340, May 1976.

[11] A. Taflove and S. C. Hagness, Computational Electrodynamics: The Finite-Difference Time-Domain Method (Third ed.), Norwood, MA: Artech House, 2005.

[12] I. E. Telatar, "Capacity of Multi-antenna Gaussian Channels," European Transactions on Telecommunications, Vol. 10, pp. 585-595, 1999.

[13] A. Scaglione, D. L. Goeckel, and J. N. Laneman, "Cooperative Communications in Mobile Ad Hoc Networks," IEEE Signal Processing Magazine, Vol. 23, No. 5, pp. 18-29, September 2006.

[14] T. L. Marzetta and B. M. Hochwald, "Capacity of a Mobile Multiple-Antenna Communication Link in Rayleigh Flat Fading," IEEE Trans. Information Theory, Vol. 45, No.1, pp. 139-157, January 1999.

[15] D. Shiu, G. J. Foschini, M. J. Gans and J. M. Kahn, "Fading Correlation and Its Effect on the Capacity of Multielement Antenna Systems," IEEE Trans. Communications, vol. 48, no. 3, pp. 502-513, March 2000.

[16] A. L. Moustakas and S. H. Simon, "On the Outage Capacity of Correlated Multiple-Path MIMO Channels," IEEE Trans. Information Theory, vol. 53, no. 11, pp. 3887-3903, November 2007.

[17] Y. Cocheril, C. Langlais, M. Berbineau and G. Moniak, "Advantages of Simple MIMO Schemes for Robust or High Data Rate Transmission Systems in Underground Tunnels," in Proc. IEEE VTC 2008-Fall, Calgary, Alberta, September 2008.

[18] S. Loyka, "Multiantenna Capacities of Waveguide and Cavity Channels," IEEE Trans. on Vehicular Technology, vol. 54, no. 3, pp. 863-872, May 2005.

[19] J. M. Molina-Garcia-Pardo, M. Lienard, P. Degauque, D. G. Dudley and L. Juan-Llacer, "Interpretation of MIMO Channel Characteristics in Rectangular Tunnels from Modal Theory," IEEE Trans. on Vehicular Technology, Vol. 57, No. 3, pp.19741979, May 2008.

[20] J. N. Laneman, D. N. C. Tse, and G. W. Wornell, "Cooperative Diversity in Wireless Networks: Efficient Protocols and Outage Behavior," IEEE Trans. Information Theory, vol. 50, no. 12, pp. 3062-3080, December 2004.

[21] A. S. Avestimehr and D. N. C. Tse, "Outage Capacity of the Fading Relay Channel in the Low-SNR Regime," IEEE Trans. Information Theory, vol. 53, no. 4, pp. 1401-1415, April 2007.

[22] Y. Shi, S. Sharma, Y. T. Hou, and S. Kompella, “Optimal Relay Assignment for Cooperative Communications," in Proc. ACM MobiHoc '08, Hong Kong SAR, China, May 2008.

[23] E. Beres and R. Adve, "Selection Cooperation in Multi-Source Cooperative Networks," IEEE Trans. Wireless Communication, vol. 7, no. 1, pp. 118-127, January 2008.

[24] A. K. sadek, Z. Han, and K. J. R. Liu, "Distributed Relay-Assignment Protocols for Coverage Expansion in Cooperative Wireless Networks,” IEEE Trans. Mobile Computing, vol. 9, no. 4, pp. 505-515, April 2010.

[25] K. D. Laakmann and W. H. Steier, "Waveguides: Characteristic Modes of Hollow Rectangular Dielectric Waveguides", Applied Optics, vol. 15, no. 5, pp. 1334-1340, May 1976.

[26] E. Limpert, W. Stahel and M. Abbt, "Log-normal Distributions across the Sciences: Keys and Clues," BioScience, vol. 51, no. 5, pp. 341-352, May 2001.

[27] N. Mehta, J. Wu, A. Molisch, and J. Zhang, “Approximating a Sum of Random Variables with a Lognormal," IEEE Trans. Wireless Communications, vol. 6, no. 7, pp. 2690-2699, July 2007.

[28] G. Strang, Linear algebra and its applications 4th ed, Belmont, CA: Thomson, Brooks/Cole, 2006.

[29] P. Billingsley, Probability and Measure (Third ed.), New York: John Wiley \& Sons, 1995.

[30] D. L. Gerlough and M. J. Huber, Traffic Flow Theory: A Monograph, Washington: National Research Council, 1975. 


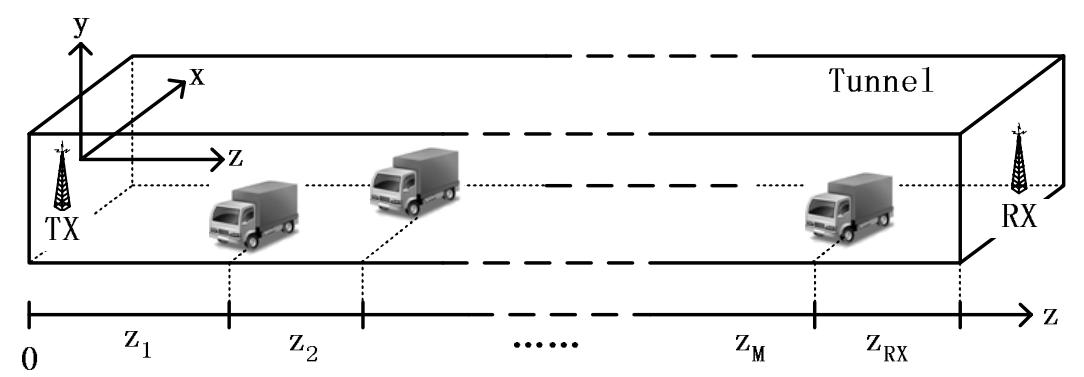

Fig. 1. Tunnel Environment

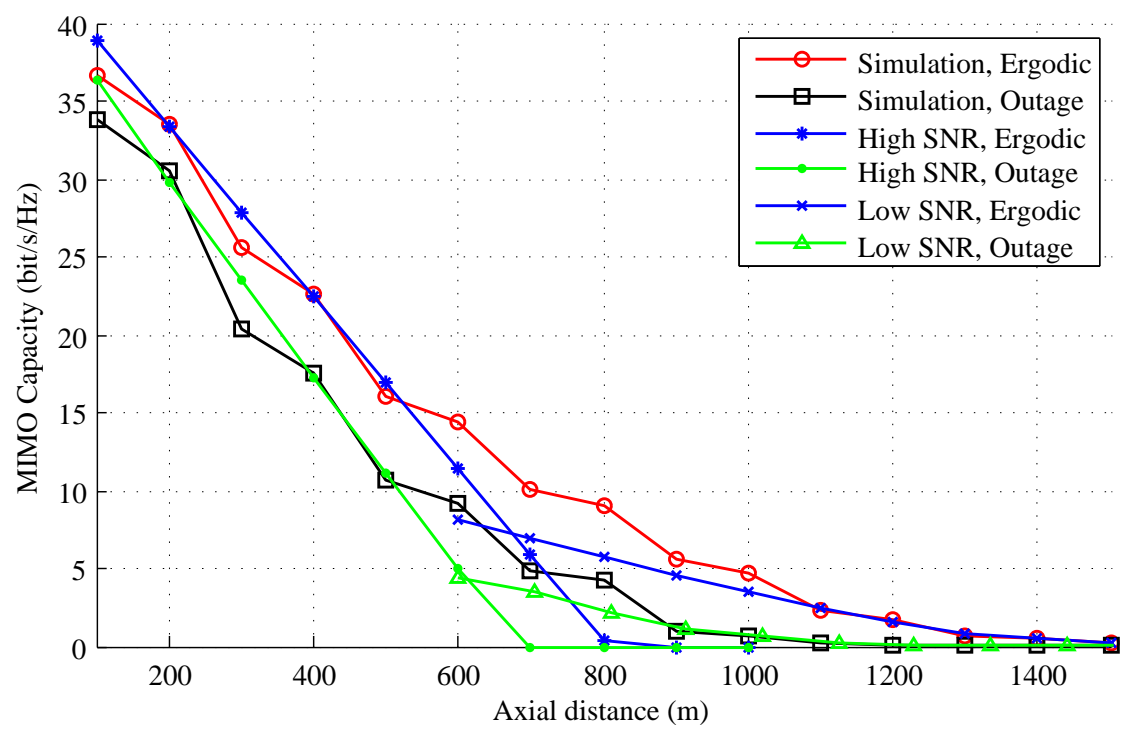

Fig. 2. Ergodic and 10\%-outage MIMO capacity as functions of the axial distance between transceivers.

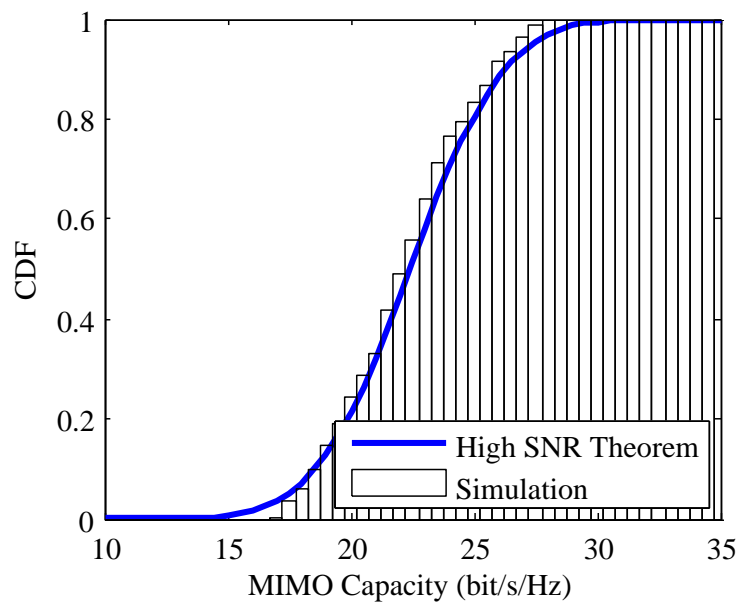

(a)

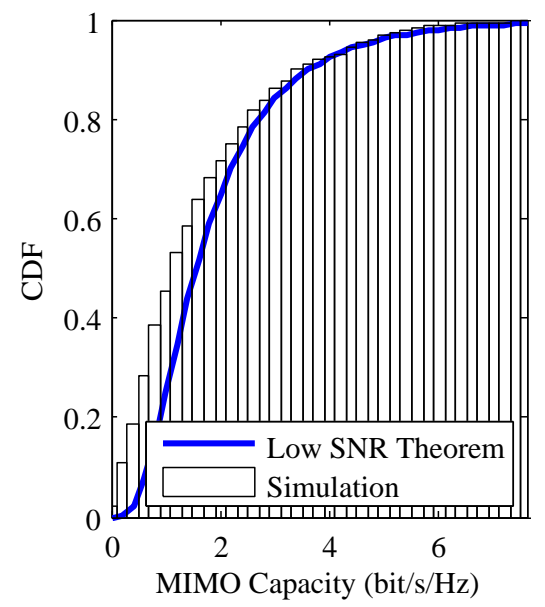

(b)

Fig. 3. CDF of MIMO capacity: (a) high SNR, (b) low SNR. 


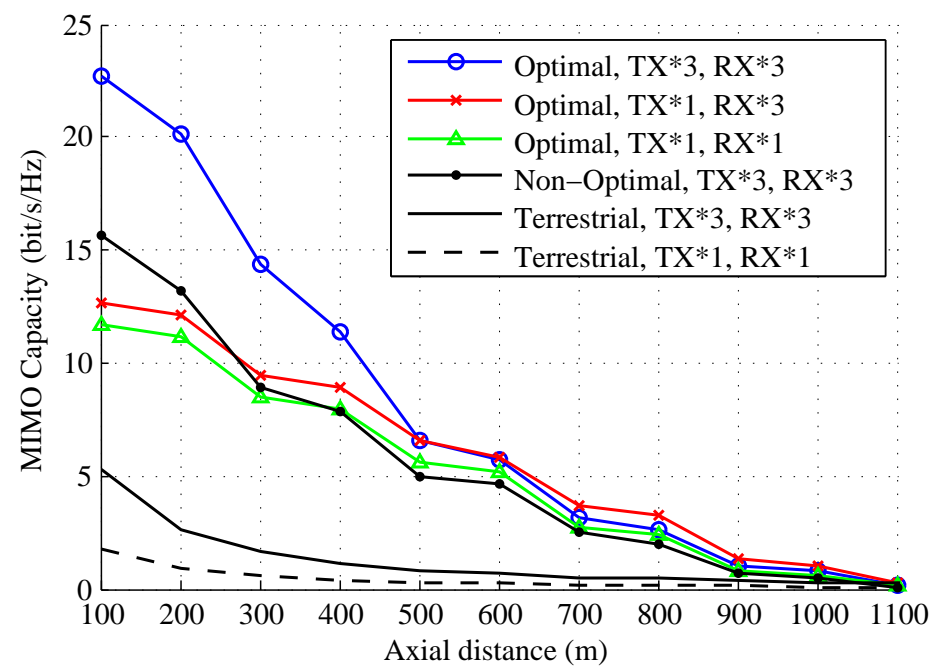

(a)

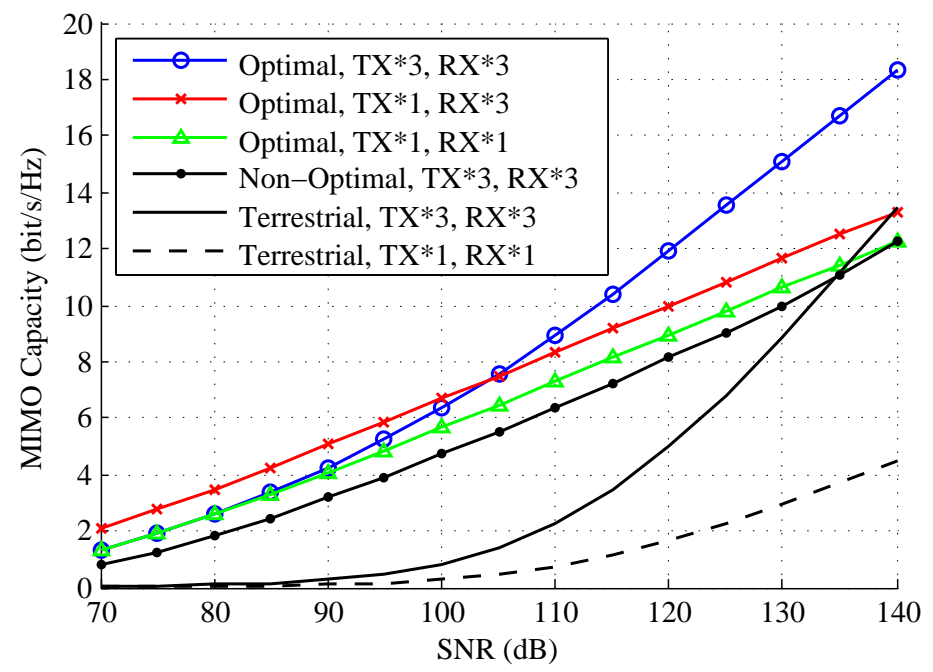

(b)

Fig. 4. 10\%-Outage MIMO capacity with different antenna geometries as a function of (a) axial distance (SNR at the transmitter is $100 \mathrm{~dB}$ ) and (b) SNR at the transmitter (axial distance is $500 \mathrm{~m}$ ). 


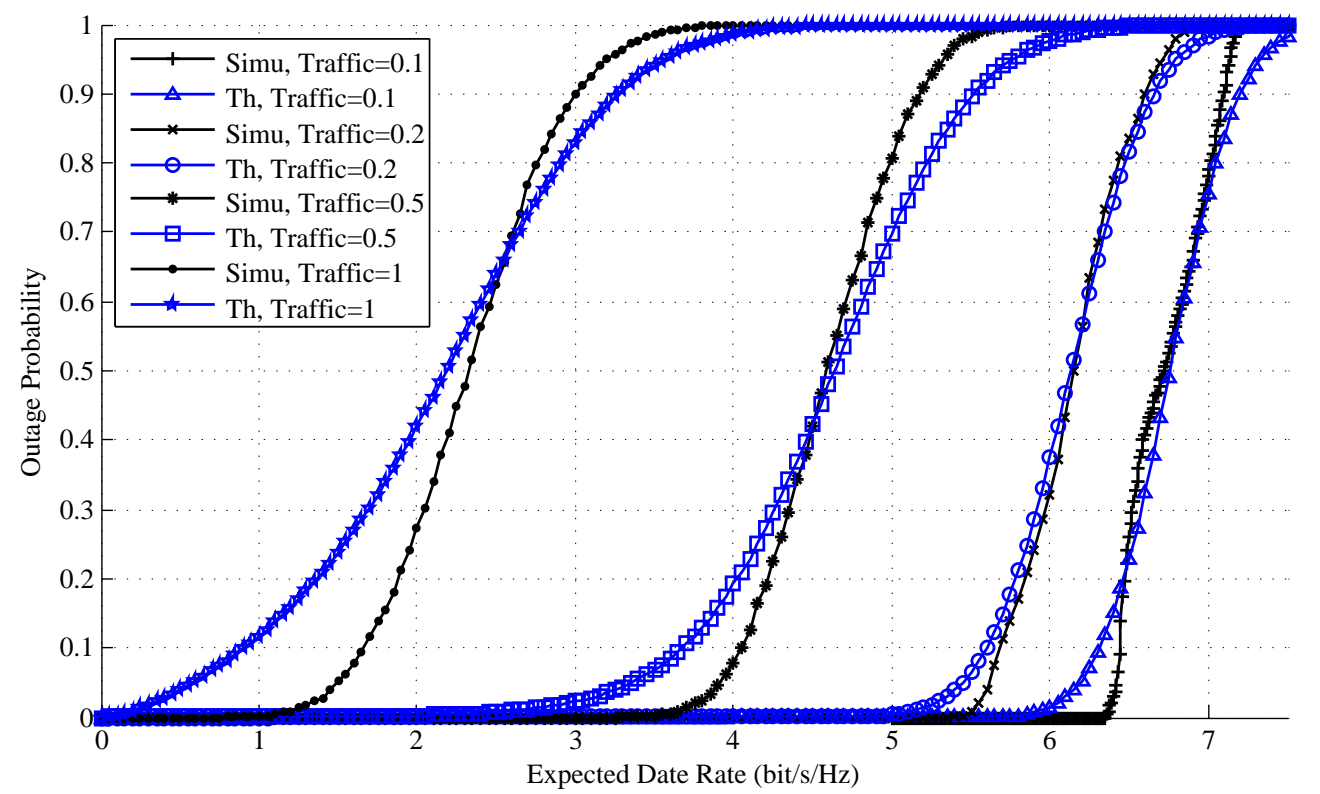

Fig. 5. Outage probabilities of cooperative communication systems with different traffic loads.

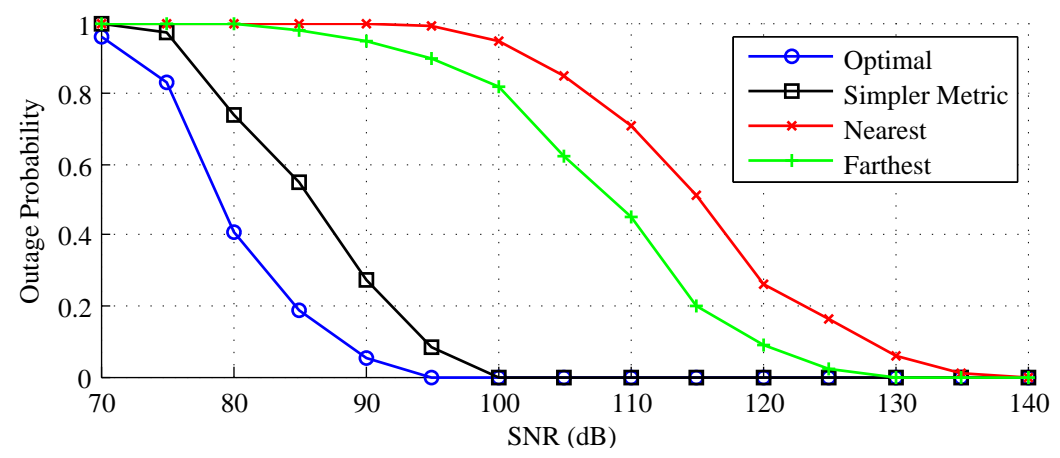

(a)

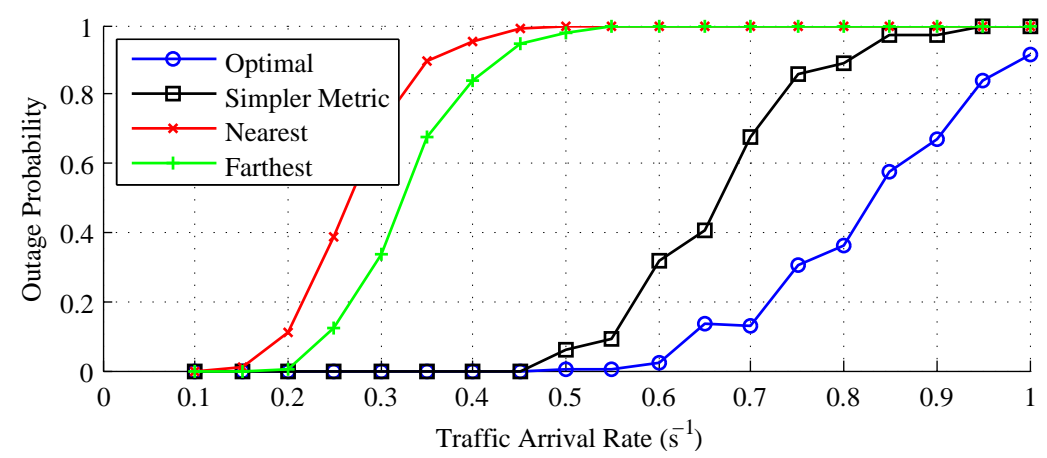

(b)

Fig. 6. Outage probability with different cooperative relay assignment strategy as functions of different (a) SNR at the transmitter and (b) traffic load. 